Supporting Information

\title{
Tuning of Optical Behavior in Monolayer and Bilayer Molybdenum Disulfide Using Hydrostatic Pressure
}

Chenkai Li ${ }^{1 \#, ~ Y a o y a o ~ L i u ~}{ }^{1}$,\#, Qishuo Yang ${ }^{1}$, Qunfei Zheng ${ }^{1}$, Zhipeng Yan ${ }^{1}$, Jun Han ${ }^{1}$, Junhao Lin ${ }^{1}$, Shanmin Wang ${ }^{1}$, Jingbo Qi ${ }^{2}$, Ying Liu ${ }^{1},{ }^{*}$ and Jinlong Zhu ${ }^{1},{ }^{*}$

1 Department of Physics, Southern University of Science and Technology, Shenzhen 518055, China.

2 State Key Laboratory of Electronic Thin Films and Integrated Devices, University of Electronic Science and Technology of China, Chengdu 611731, China

\# Authors contributed equally to this work.

* Corresponding authors: Ying Liu: liuy37@sustech.edu.cn; Jinlong Zhu: zhujl@sustech.edu.cn. 

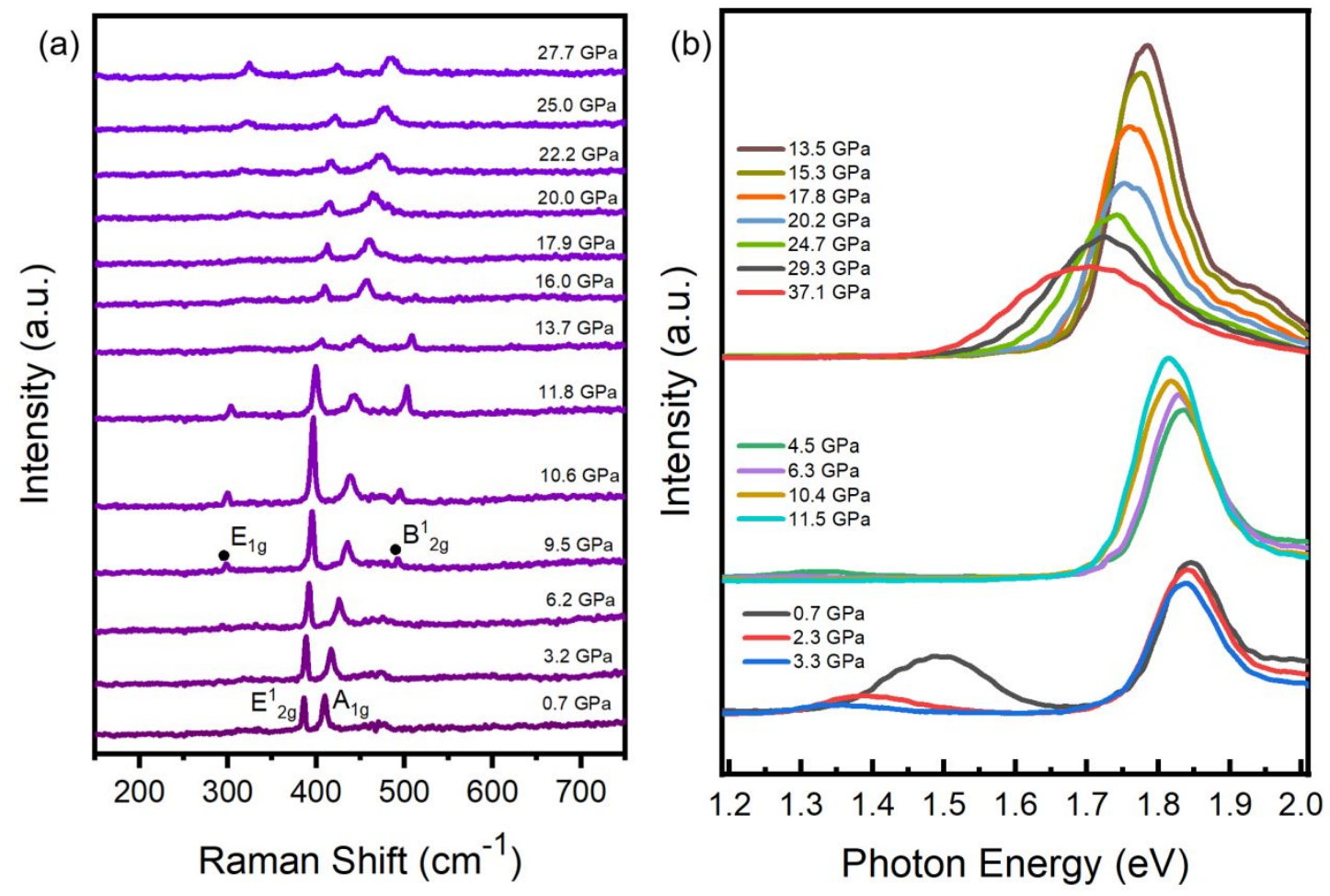

Figure S1 (a) Raman and (b) PL spectra of bilayer $\mathrm{MoS}_{2}$ at different pressure with $\mathrm{He}$ as PTM.
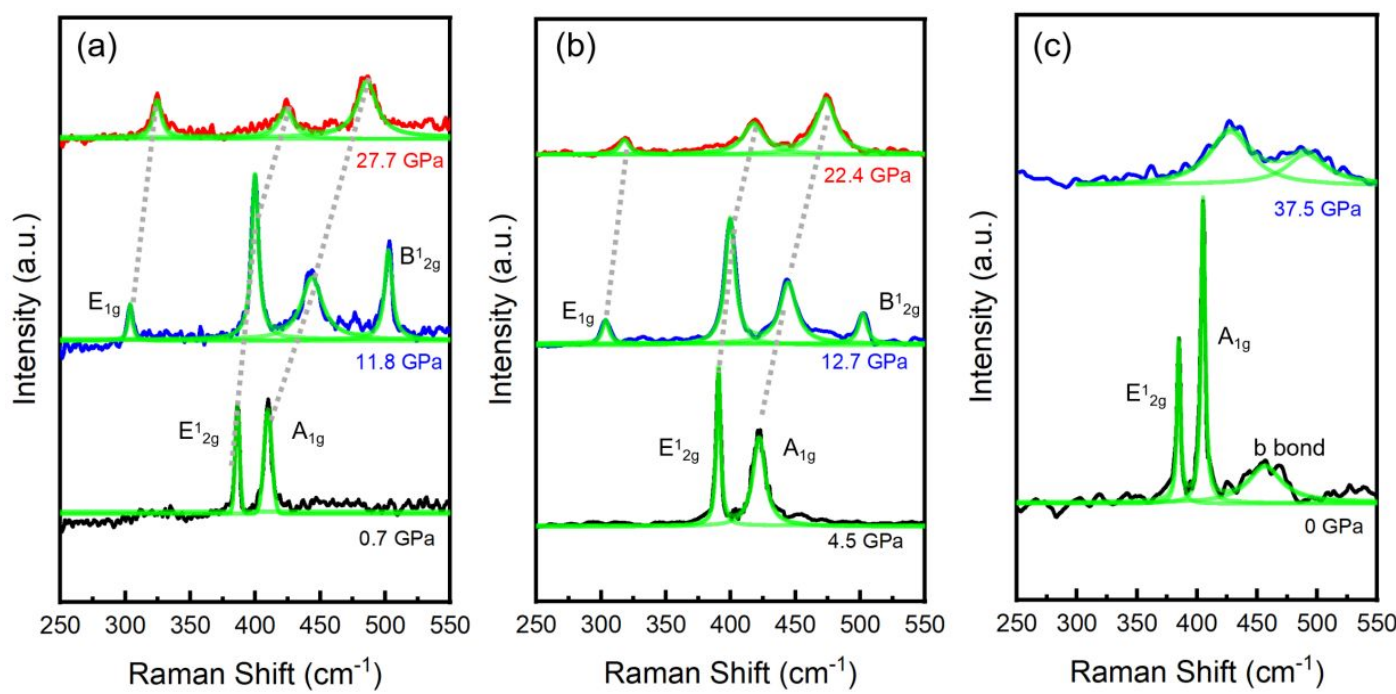

Figure S2 Raman fitting examples of (a) bilayer on $\mathrm{He}$, (b) bilayer on $\mathrm{Ar}$, (c) monolayer on $\mathrm{Ar}$ at selected representative pressures (the green lines are fitting line, the gray dash lines are guiding for the eyes). 


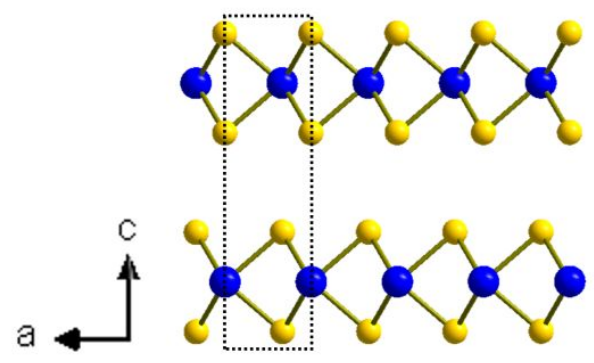

$2 \mathrm{H}_{c}$

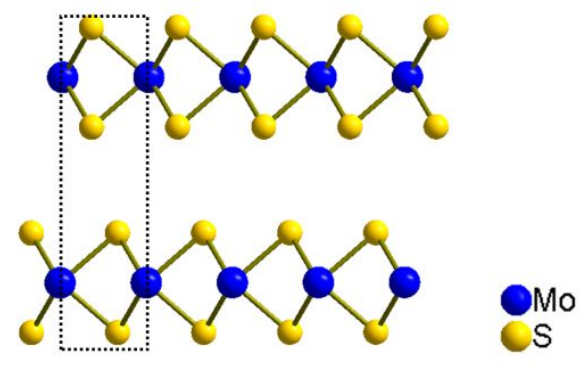

$2 \mathrm{H}_{\mathrm{a}}$

Figure S3 Schematics of $2 \mathrm{H}_{\mathrm{c}}$ and $2 \mathrm{H}_{\mathrm{a}}$ structures.

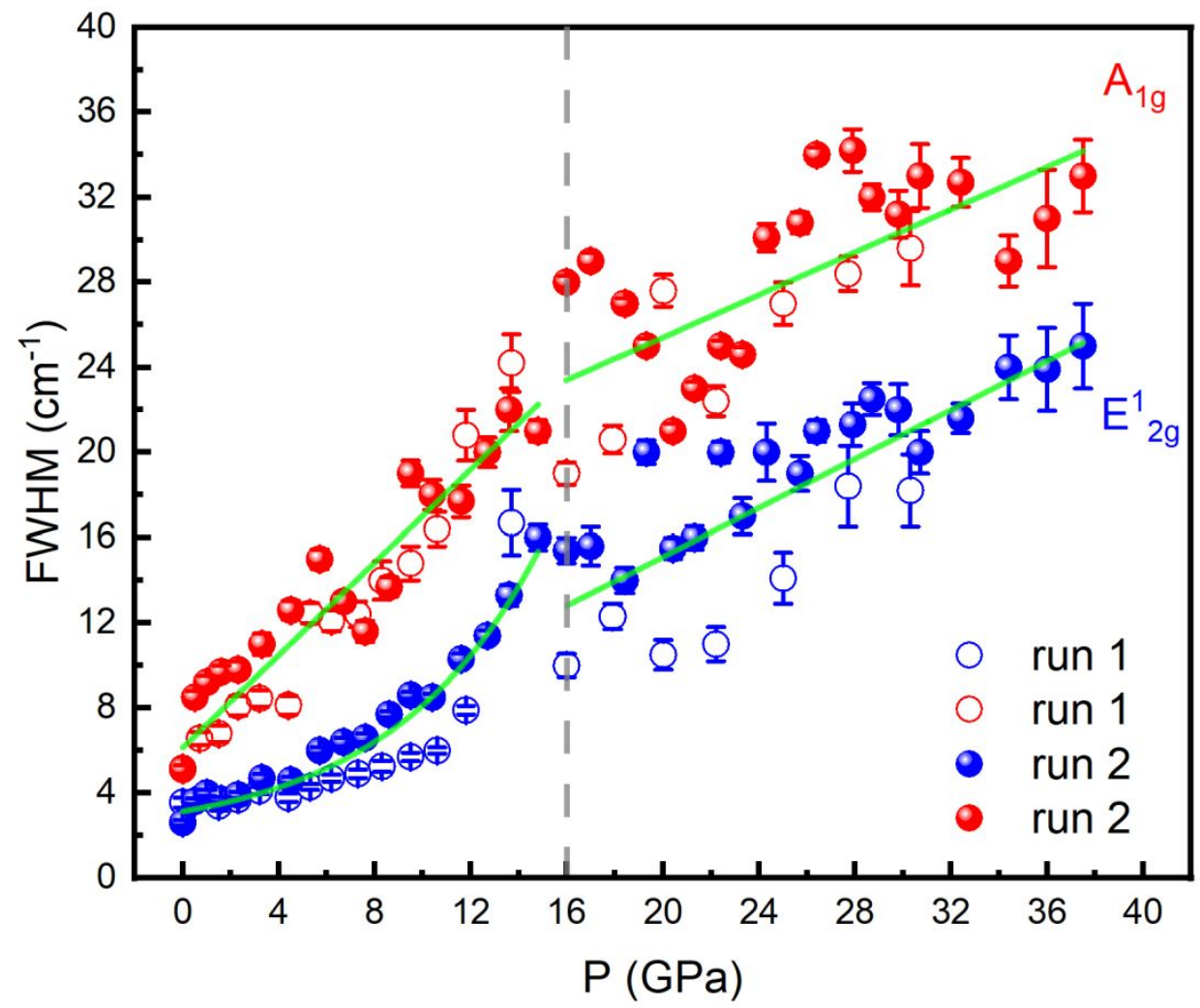

Figure S4 Pressure dependence of FWHM of $A_{1 g}$ and $E_{2 g}^{1}$ of bilayer $M_{0} S_{2}$, run1 uses He as PTM and run 2 uses Ar as PTM (the green lines are guiding for the eyes). 


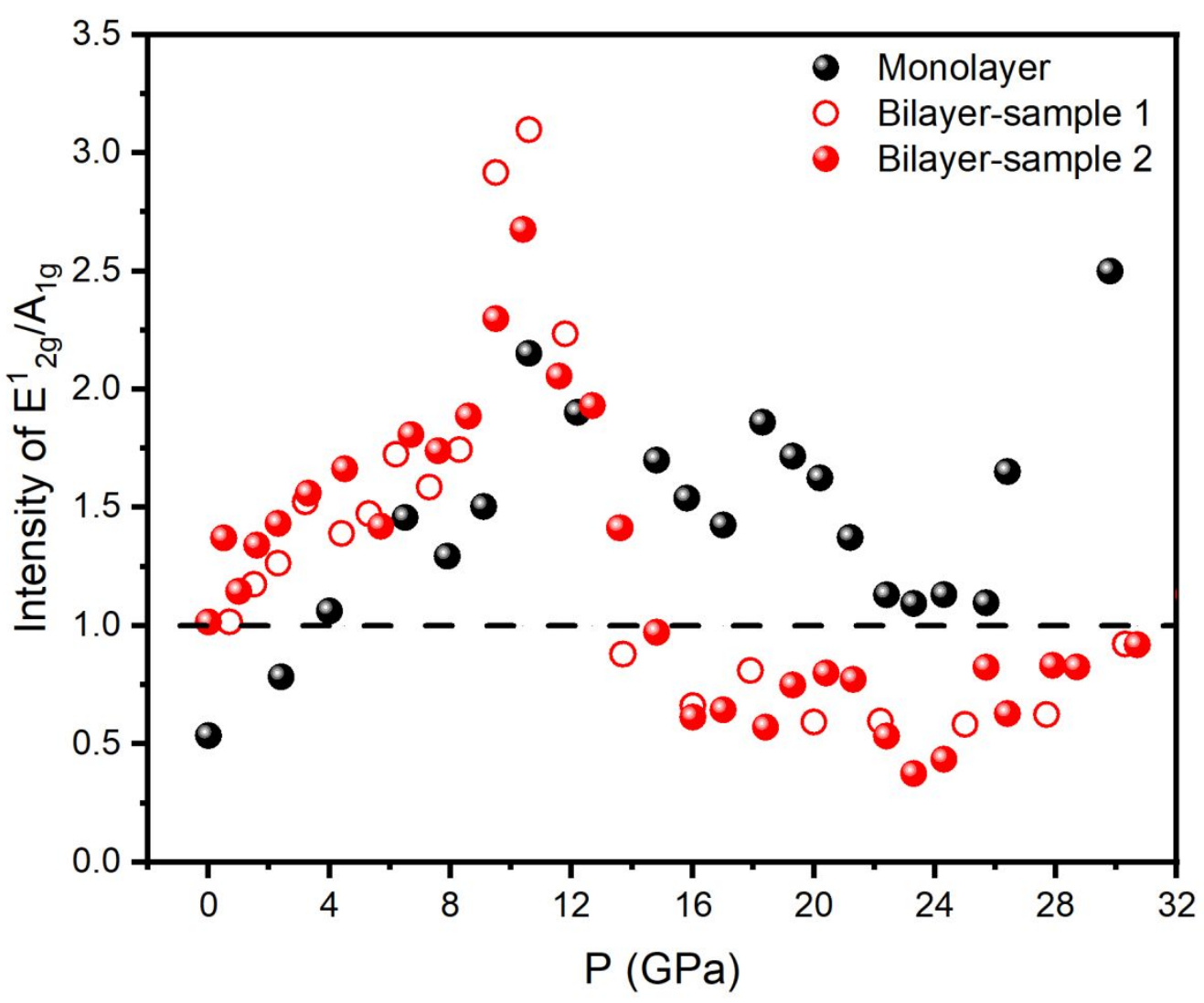

Figure $\mathrm{S} 5$ The ratio of intensity of $\mathrm{E}^{1}{ }_{2 \mathrm{~g}}$ and $\mathrm{A}_{1 \mathrm{~g}}$ modes of bilayer and monolayer $\mathrm{MoS}_{2}$ under different pressures. Sample 1 uses He as PTM and sample 2 uses Ar as PTM.

For bilayer $\mathrm{MoS}_{2}$, the intensity of $\mathrm{E}^{1}{ }_{2 g}$ mode was higher than $\mathrm{A}_{1 \mathrm{~g}}$ mode before about $14 \mathrm{GPa}$ and vice versa after about $14 \mathrm{GPa}$. Different from bilayer, the $E^{1}{ }_{2 g}$ mode is dominant always for the monolayer. 


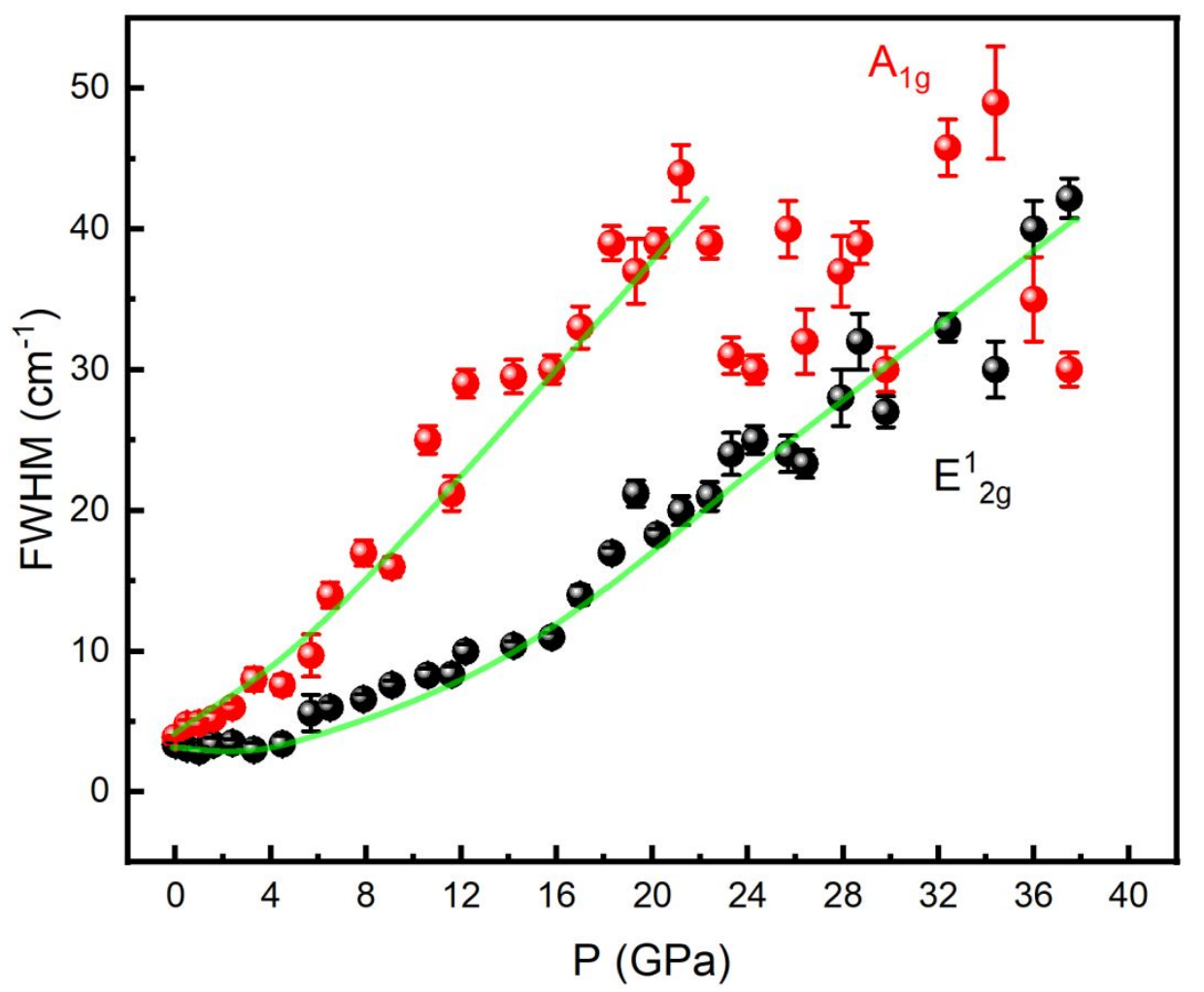

Figure S6 Pressure dependence of FWHM of $A_{1 g}$ and $E^{1}{ }_{2 g}$ of monolayer $\mathrm{MoS}_{2}$ (the green lines are guiding for the eyes).
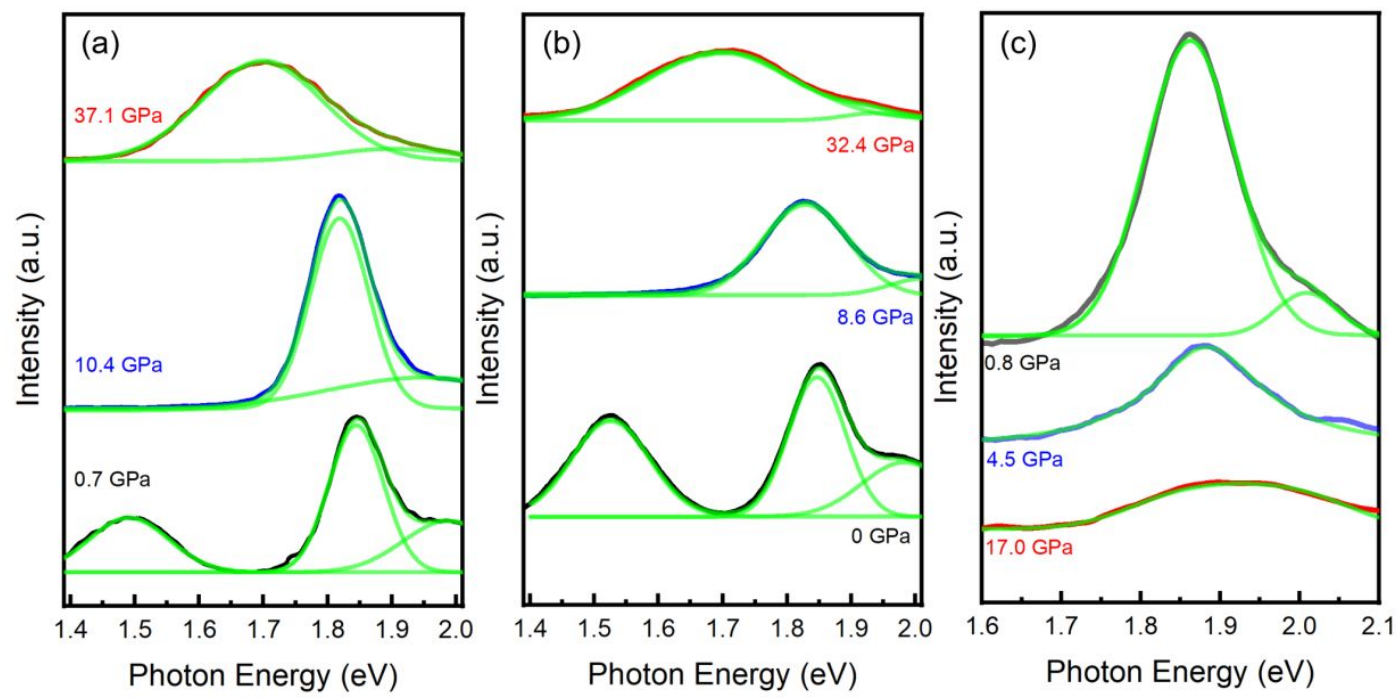

Figure S7 PL fitting examples of (a) bilayer on $\mathrm{He}$, (b) bilayer on $\mathrm{Ar}$, (c) monolayer on $\mathrm{Ar}$ at selected representative pressures (the green lines are fitting line). 

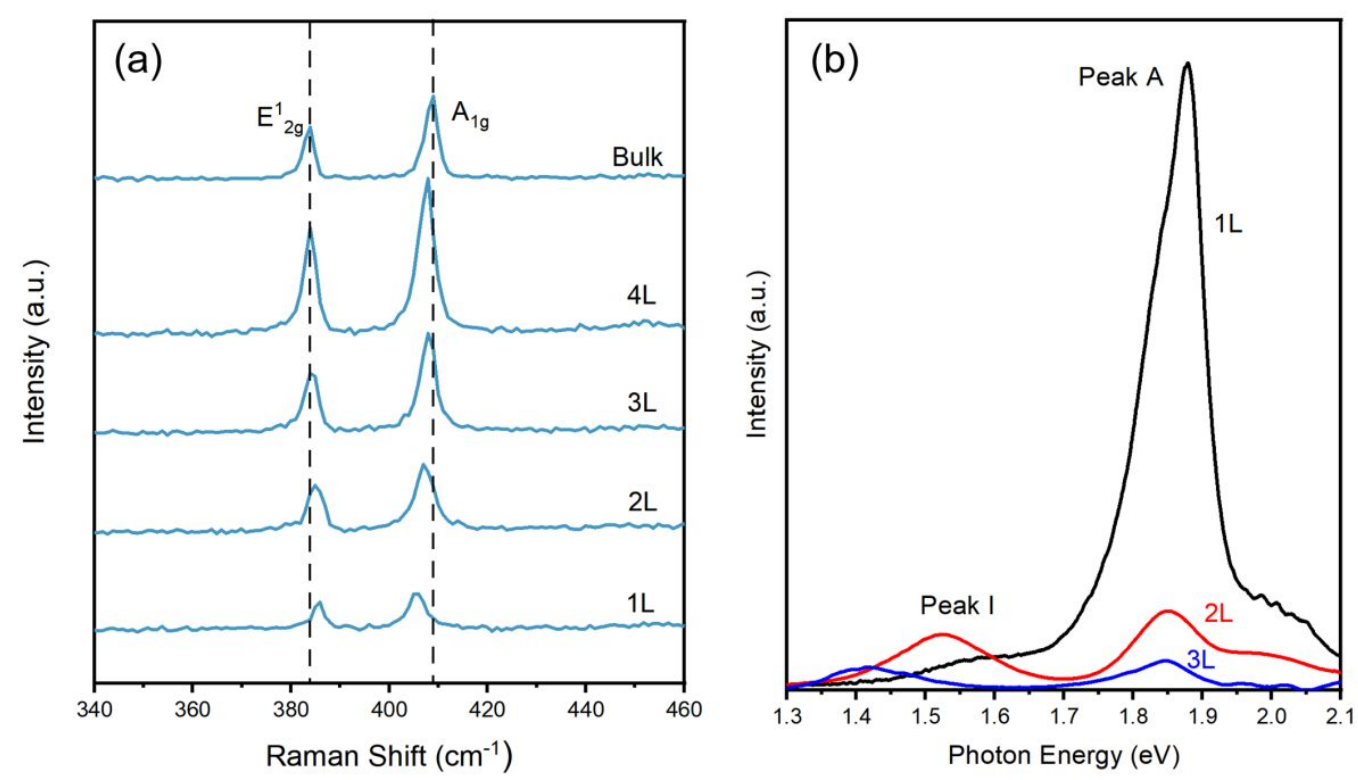

Figure S8 (a) Raman spectra of bulk, 4L-, 3L-, 2L- and 1L-MoS ${ }_{2}$ at ambient conditions; (b) PL spectra of 3L-, 2L- and 1L-MoS 2 at ambient conditions.

The frequency difference between the $E_{2 g}^{1}$ and $A_{1 g}$ modes, the intensity of $P L$ peaks and band gap are the factors to identify the layer numbers of fewlayer $\mathrm{MoS}_{2}$. As the decrease of a layer number, the frequency difference decreases. For bulk, 4L-, 3L-, 2L- and $1 \mathrm{~L}-\mathrm{MoS}_{2}$, the frequency difference was about $25 \mathrm{~cm}^{-1}, 24 \mathrm{~cm}^{-1}, 23 \mathrm{~cm}^{-1}, 22 \mathrm{~cm}^{-1}$ and $19 \mathrm{~cm}^{-1}$, respectively. Meanwhile, the intensity of PL peaks decreases and band gap decreases. For $4 \mathrm{~L}-\mathrm{MoS}_{2}$, the $\mathrm{PL}$ is hard to detect. The band gaps are about $1.41 \mathrm{eV}, 1.53 \mathrm{eV}$ and 1.88 $\mathrm{eV}$ for $3 \mathrm{~L}-, 2 \mathrm{~L}-$ and $1 \mathrm{~L}-\mathrm{MoS}_{2}$, respectively. The dramatically increase of $\mathrm{PL}$ is a key to identify monolayer $\mathrm{MoS}_{2}$. All results are consistent with previous studies $^{1-6}$. 


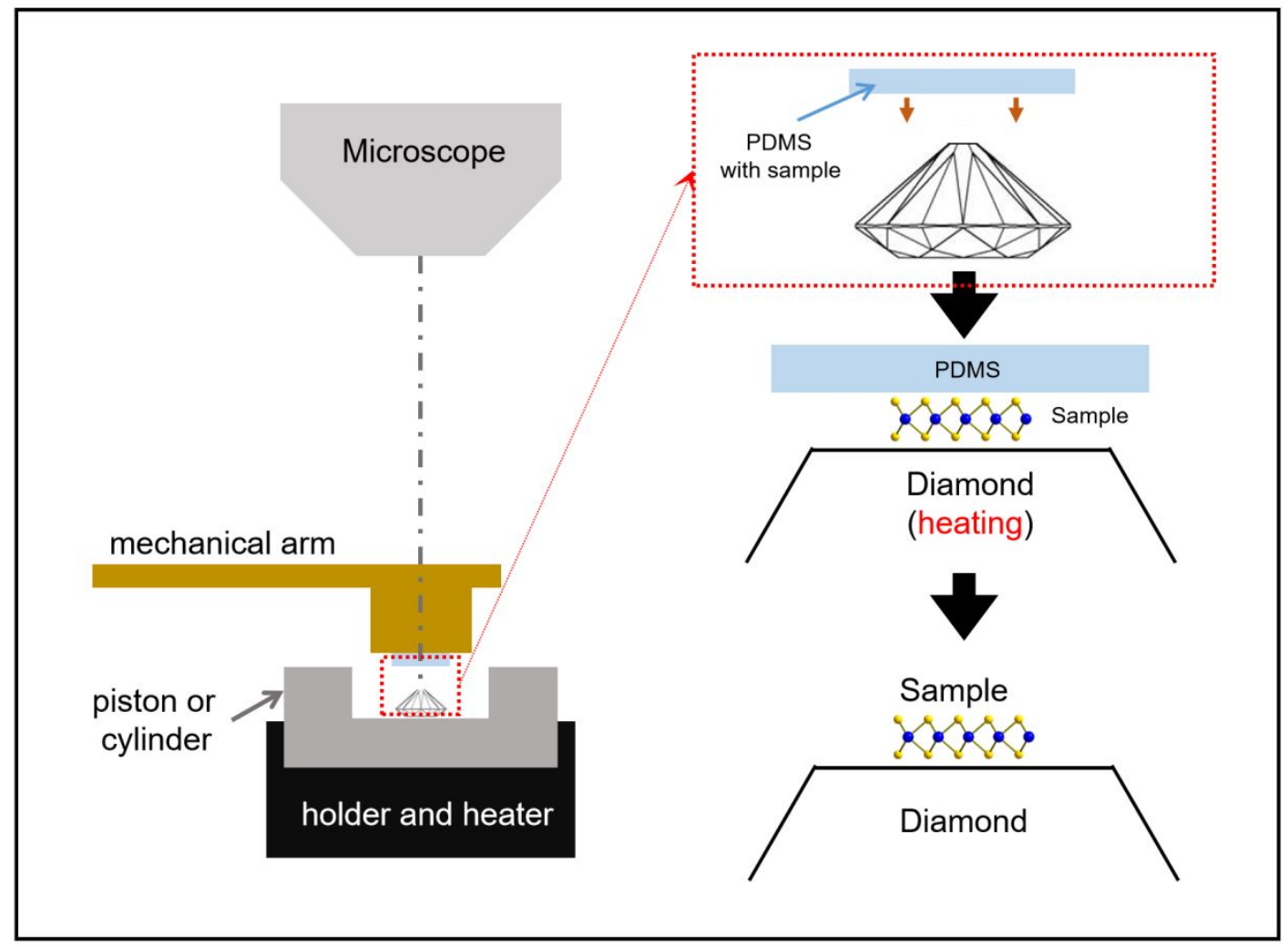

Figure S9 The method to transfer the films from the PDMS to the culet of DAC.

As shown in Figure S9, the $\mathrm{MoS}_{2}$ films on PDMS will touch the culet of DAC horizontally and slowly with help of microscope and mechanical arm. Then the heater heats the piston or cylinder to just below 80 degrees. As the heat conducts through the diamond anvil to the PDMS, the interaction between the films and the PDMS will weaken. Further, under the influence of coupling between the films and the culet, the films will leave the PDMS and fall on the culet of DAC. 


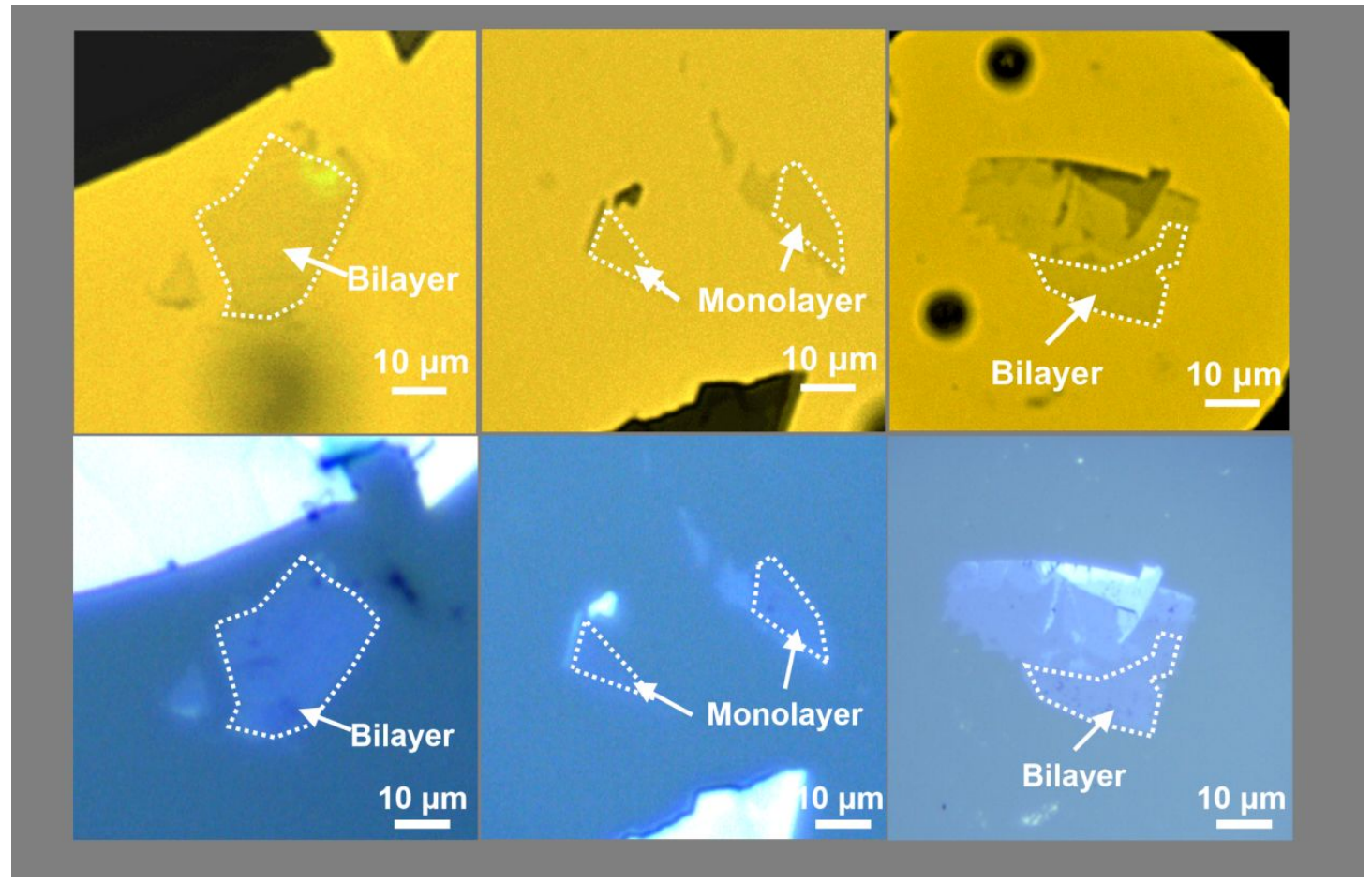

Figure S10 The $\mathrm{MoS}_{2}$ films in DAC with transmission light (up) and reflected light (down). The scales and the layer number with white dashed boxes showing the boundary of monolayer or bilayer are added.

\section{References}

(1) Li, F.; Yan, Y.; Han, B.; Li, L.; Huang, X.; Yao, M.; Gong, Y.; Jin, X.; Liu, B.; Zhu, C., et al. pressure confinement effect in mos2 monolayers. Nanoscale 2015, 7, 9075-9082.

(2) Dou, X.; Ding, K.; Jiang, D.; Sun, B. tuning and identification of interband transitions in monolayer and bilayer molybdenum disulfide using hydrostatic pressure. ACS Nano 2014, 8, 7458-7464.

(3) Wang, Q. H.; Kalantar-Zadeh, K.; Kis, A.; Coleman, J. N.; Strano, M. S. electronics and optoelectronics of two-dimensional transition metal dichalcogenides. Nature nanotech 2012, 7, 699-712.

(4) Lee, C.; Yan, H.; Brus, L. E.; Heinz, T. F.; Hone, J.; Ryu, S. anomalous lattice vibrations of single-and few-layer mos2. ACS nano 2010, 4, 2695-2700. 
(5) Chakraborty, B.; Matte, H.; Sood, A. K.; Rao, C. layer-dependent resonant raman scattering of a few layer mos2. J RAMAN 2013, 44.1: 92-96.

(6) Mak, K. F.; Lee, C.; Hone, J.; Shan, J.; Heinz, T. F. atomically thin mos2: a new direct-gap semiconductor. Phys. Rev. Lett. 2010, 105, 136805 